\title{
Goat milk consumption modulates liver divalent metal transporter 1 (DMT1) expression and serum hepcidin during Fe repletion in Fe-deficiency anemia
}

\author{
J. Díaz-Castro, ${ }^{*}$ M. Pulido,† M. J. M. Alférez, ${ }^{*}$ J. J. Ochoa, ${ }^{*}$ E. Rivas, ${ }^{*}$ S. Hijano, ${ }^{*}$ and I. López-Aliaga*¹ \\ *Department of Physiology, and \\ †Department of Biochemistry and Molecular Biology II, Faculty of Pharmacy, University of Granada and Institute of Nutrition and Food Technology, \\ E-18071 Granada, Spain
}

\section{ABSTRACT}

Iron deficiency is the most prevalent micronutrient deficiency worldwide. In spite of the crucial role of hepatocyte divalent metal transporter 1 (DMT1) and hepcidin in $\mathrm{Fe}$ metabolism, to date, no studies have directly tested the role of these proteins in liver $\mathrm{Fe}$ metabolism during Fe repletion after induced Fedeficiency anemia. Therefore, the aim of the current study was to assess the effect of goat or cow milk-based diets on Fe metabolism in one of the main body storage organs, the liver, during the course of Fe repletion with goat or cow milk-based diets in anemic rats. Animals were placed on a preexperimental period of $40 \mathrm{~d}$, a control group receiving a normal-Fe diet and the Fedeficient group receiving a low-Fe diet $(5 \mathrm{mg}$ of $\mathrm{Fe} / \mathrm{kg}$ of diet). Rats were fed for $30 \mathrm{~d}$ with goat or cow milkbased diets with normal Fe content (45 mg of Fe/ $\mathrm{kg}$ of diet). The hematological parameters, serum hepcidin, hepatosomatic index, liver Fe content, and liver DMT1 expression were determined. During the recovery of the anemia with milk-based diets, the restoration of liver Fe content and hematological parameters, especially with goat milk, increased the red blood cell count, favoring the oxygen supply and weight gain. Moreover, goat milk consumption potentiates liver DMT1 expression, enhancing Fe metabolism and storage. In addition, the increase in serum hepcidin in anemic rats observed in the current study also explains and supports the higher liver Fe content after supplying goat milk, because it blocks the liberation of Fe from hepatocytes, increasing its storage in liver.

Key words: Fe-deficiency anemia, divalent metal transporter 1, serum hepcidin, goat and cow milk

Received July 12, 2013.

Accepted October 1, 2013.

${ }^{1}$ Corresponding author: milopez@ugr.es

\section{INTRODUCTION}

According to the World Health Organization, anemia affects almost 2 billion people worldwide, with Fe deficiency anemia accounting for 75 to $80 \%$ of the total burden (WHO, 2008). However, it is being recognized that current recommendations may be higher than necessary, and higher Fe doses have a high incidence of undesirable side effects, including $\mathrm{Fe}$ overload and gastrointestinal disorders (Ekström et al., 2002). Approximately 25 to $50 \%$ of body storage $\mathrm{Fe}$ is found in the liver, mainly in hepatocytes and Kupffer cells. In Fe-overload disorders, such as hereditary hemochromatosis, hepatic Fe stores increase progressively, with Fe being deposited predominantly in hepatocytes (Pietrangelo, 2010). Divalent metal transporter 1 (DMT1; also called Nramp2 and DCT1) was first identified as a transmembrane Fe-transport protein by Gunshin et al. (1997) in the enterocytes, and because DMT1 is also expressed in the liver, it plays a crucial role in $\mathrm{Fe}$ metabolism (Trinder et al., 2000; Anderson and Vulpe, 2009), either through the uptake of transferrin-bound $\mathrm{Fe}$ or non-transferrin-bound $\mathrm{Fe}$, which appears in the plasma during $\mathrm{Fe}$ overload (Grootveld et al., 1989). Notably, DMT-1 is ubiquitously expressed in the body and is involved (in ways not yet fully elucidated) in $\mathrm{Fe}$ excretion and (or) reabsorption by the kidneys and liver in erythropoiesis and in tissue Fe distribution (Gunshin et al., 2005). Thus, dietary modulation of DMT-1 function may affect whole-body Fe homeostasis and hence hematological status.

Another key protein in $\mathrm{Fe}$ metabolism is the mature 25-AA hepatic peptide hepcidin, which has been identified as the master regulator of $\mathrm{Fe}$ metabolism (Viatte and Vaulont, 2009; Tselepis et al., 2010). It is also clear that several pathways exist involved in the regulation of this peptide hormone, including inflammation, infection, erythropoietic demand, hypoxia, and body Fe status (Zhang and Enns, 2009). Hepcidin works by blocking the entry of Fe into the circulation from the gut and the macrophages by binding to the Fe transporter ferroportin, resulting in its ubiquitylation 
and degradation (De Domenico et al., 2007). Hepcidin deficiency is the cause of Fe overload in hereditary hemochromatosis, whereas hepcidin excess is associated with anemia of inflammation, chronic kidney disease, and Fe-deficiency anemia (Ganz, 2011).

On the other hand, we have previously reported (Alférez et al., 2006; Nestares et al., 2008) that goat milk improves Fe metabolism, enhancing Fe digestive and metabolic utilization in both control and anemic rats, increasing $\mathrm{Fe}$ deposits in target organs, favoring the recovery of hematological parameters and minimizing interactions between dietary divalent cations such as $\mathrm{Ca}$ and $\mathrm{Mg}$. In spite of the crucial role of hepatocyte DMT1 and hepcidin in liver Fe metabolism, to date, no studies have directly tested the role of these proteins in liver Fe metabolism during Fe repletion after induced Fe-deficiency anemia. Taking into account all these considerations, the present study was carried out to evaluate the hematological status and to know how it is affected by Fe metabolism in the main body storage organ, the liver. The study was carried out during the course of the Fe deficiency and Fe repletion with goat or cow milk-based diets, assessing 2 of the key regulators of Fe metabolism, hepcidin and DMT1, as well as systemic Fe status.

\section{MATERIALS AND METHODS}

\section{Animals}

Sixty male Wistar albino breed rats (21 d of age and weighing about $42 \pm 5 \mathrm{~g}$ ), purchased from the University of Granada Laboratory Animal Service (Granada, Spain) were used during the study. All animal care procedures and experimental protocols were approved by the Ethics Committee of the University of Granada according to the European Community guidelines.

\section{Experimental Design and Diets}

At the beginning of the study, the rats were randomly divided into 2 groups: the control group receiving a normal-Fe diet $(45 \mathrm{mg} / \mathrm{kg}$; Reeves et al., 1993) and the anemic group receiving a low-Fe diet $(5 \mathrm{mg} / \mathrm{kg})$ according to a technique developed previously by us (Pallarés et al., 1993; Table 1).

During the course of the study, the animals were kept at an automatically controlled temperature $\left(22-23^{\circ} \mathrm{C}\right)$, humidity (55-65\%), and a 12-h light-dark cycle (0900 to $2100 \mathrm{~h}$ ). Mineral-free water was available ad libitum, but the diet intake was controlled, pair feeding all the animals to avoid any differences due to the intake. The diet supplied was calculated as $80 \%$ of the average intake on each experimental period (EP). On d 40 of the preexperimental period (PEP), 10 rats per group (10 controls and 10 anemic rats) after fasting overnight, were weighed and anesthetized by intraperitoneal injection of $5 \mathrm{mg} / 100 \mathrm{~g}$ of BW of sodium pentobarbital (Sigma-Aldrich, St Louis, MO). Animals were carefully weighed and after median laparotomy, the rats were totally bled out by cannulation of the abdominal aorta. Aliquots of blood were obtained with EDTA as anticoagulant to measure the hematological parameters. The remaining blood was centrifuged at $1,500 \times \mathrm{g}$ for $15 \mathrm{~min}$ at $4^{\circ} \mathrm{C}$ without anticoagulant to separate the red blood cells (RBC) from the serum and subsequent analysis of Fe, ferritin, total Fe-binding capacity (TIBC), and hepcidin. The livers were removed, washed with icecold saline solution $(0.9 \% \mathrm{NaCl}$, wt/vol $)$, weighed, and stored immediately at $-20^{\circ} \mathrm{C}$ until Fe analysis.

After induction of the anemia (d 40 of the study), the remaining animals $(\mathrm{n}=40)$ were then placed on an EP in which the control and anemic groups (control rats fed a goat milk diet, anemic rats fed a goat milk diet, control rats fed a cow milk diet, and anemic rats fed a cow milk diet) were further fed for $30 \mathrm{~d}$ a goat or cow milk-based diet with normal Fe content (45 mg/ $\mathrm{kg}$ ), prepared with goat (Murciano-Granadina breed) or cow (Holstein breed) skimmed milk powder $(20 \%$

Table 1. Composition of the experimental diets

\begin{tabular}{lc}
\hline Component & $\begin{array}{c}\text { Amount, } \\
\mathrm{g} / \mathrm{kg} \text { of diet }\end{array}$ \\
\hline Preexperimental period standard (non-milk) diet & \\
Casein & 200 \\
Lactose & 0 \\
Fat (virgin olive oil) & 100 \\
Wheat starch & 500 \\
Constant ingredients ${ }^{2}$ & 200 \\
Experimental period & \\
Goat milk-based diet ${ }^{3}$ & \\
Goat milk protein & 205 \\
Goat milk lactose & 290 \\
Goat milk fat & 100 \\
Wheat starch & 205 \\
Constant ingredients ${ }^{2}$ & 200 \\
Cow milk-based diet ${ }^{3}$ & \\
Cow milk protein & 204 \\
Cow milk lactose & 295 \\
Cow milk fat & 100 \\
Wheat starch & 201 \\
Constant ingredients ${ }^{2}$ & 200 \\
\hline
\end{tabular}

${ }^{1}$ The diets were prepared according to the recommendations of the American Institute of Nutrition AIN-93 diet for control rats (45 mg of $\mathrm{Fe} / \mathrm{kg}$ of diet; Reeves et al., 1993), or with low Fe content (5 mg of Fe/ $\mathrm{kg}$ of diet; Pallarés et al., 1993) for Fe-deficient groups.

${ }^{2}$ The constant ingredients consisted of $(\mathrm{g} / \mathrm{kg}$ of diet) fiber (micronized cellulose; 50), sucrose (100), choline chloride (2.5), L-cystine (3), mineral premix (35), and vitamin premix (10).

${ }^{3}$ Specific vitamin and mineral premix supplements for goat and cow milk-based diets were formulated, taking into account the mineral and vitamin contents of the skim milk powder supplied to meet the recommendations of the AIN-93 (45 mg of Fe/kg of diet; Reeves et al., 1993). 
protein) and $10 \%$ fat, obtained by purification from goat or cow milk fat (Table 1$)$. The Fe content $(\mathrm{mg} / \mathrm{kg})$ in the diets by analysis was: 43.7 (goat milk-based diet) and 42.9 (cow milk-based diet). On d 70 of the study, animals were weighed and anesthetized and totally bled out by cannulation of the abdominal aorta. The blood was collected to obtain serum and later the livers were also removed, washed with ice-cold saline solution $(0.9 \%$ $\mathrm{NaCl}$, wt/vol), weighed, and split into 2 homogeneous portions, one to determine Fe content, which was frozen at $-20^{\circ} \mathrm{C}$ and the other one to prepare homogenates freshly, on the same day.

\section{Fe Determination in the Diets and Liver}

Samples of diets and liver (1 $\mathrm{g}$ of $\mathrm{DW})$ were previously mineralized by a wet method in a sand bath ( J. P. Selecta SA, Barcelona, Spain), following the method described previously (Díaz-Castro et al., 2008). Iron concentrations in the different diets were determined by atomic absorption spectrophotometry (PerkinElmer Analyst 1100B spectrometer with WinLab32 for AA software; PerkinElmer, Überlingen, Germany). To calibrate the measurements, samples of lyophilized bovine liver (certified reference material BCR 185; Community Bureau of References, Brussels, Belgium) were simultaneously used to check the Fe recovery.

\section{Hematological Test}

Hemoglobin, RBC, and hematocrit concentrations, and mean corpuscular volume and platelet concentration were measured using an automated hematology analyzer Sysmex K-1000D (Sysmex Corp., Tokyo, Japan).

\section{Serum Ferritin}

Serum ferritin concentration was determined using the Rat Ferritin ELISA Kit (BioVendor GmbH, Heidelberg, Germany). The absorbance of the reaction was read at $450 \mathrm{~nm}$ using a microplate reader (Bio-Rad Laboratories Inc., Hercules, CA). Color intensity developed was inversely proportional to the concentration of serum ferritin.

\section{Serum Fe, TIBC, and Transferrin Saturation}

To calculate the rate of transferrin saturation, serum $\mathrm{Fe}$ concentration and TIBC were determined using Sigma Diagnostics Iron and TIBC reagents (SigmaAldrich). The absorbance of samples was read at 550 $\mathrm{nm}$ on a microplate reader (Bio-Rad Laboratories Inc.). The percentage of transferrin saturation was calculated from the following equation:
Transferrin saturation $(\%)=$ serum Fe concentration

$$
(\mu \mathrm{g} / \mathrm{L}) / \mathrm{TIBC}(\mu \mathrm{g} / \mathrm{L}) \times 100 .
$$

\section{Serum Hepcidin}

Hepcidin-25 concentration was determined using a DRG ELISA Kit (DRG Instruments GmbH, Marburg, Germany). The microtiter wells were coated with a monoclonal (mouse) antibody directed toward an antigenic site of the hepcidin-25 molecule. Endogenous hepcidin-25 of a sample competed with a hepcidin25-biotin conjugate for binding to the coated antibody. After incubation, the unbound conjugate was washed off and a streptavidin-peroxidase enzyme complex was added to each well. After incubation, unbound enzyme complex was washed off and substrate solution was added. The blue color development was stopped after a short incubation time, turning the color from blue to yellow. The microplate was read at $450 \mathrm{~nm}$ on a microplate reader (Bio-Rad Laboratories Inc.) and the intensity of color developed was reverse proportional to the concentration of hepcidin in the sample. Results were expressed in nanograms per milliliter of serum.

\section{Western Blotting and Immunocytochemistry}

Finely chopped liver samples were added to a glass Potter-Elvehjem apparatus on ice and whole-cell homogenates were extracted from rat liver tissue samples by homogenization in $500 \mu \mathrm{L}$ of radioimmunoprecipitation assay (RIPA) buffer (10 $\mathrm{m} M$ Tris, $150 \mathrm{mM} \mathrm{NaCl}$, $1 \mathrm{~m} M$ EDTA, $1 \%$ Nonidet P-40, and 0.1\% SDS) and protease inhibitor mixture (1:200 dilution; SigmaAldrich). The homogenates were centrifuged at 1,000 $\times g$ for 5 min at $4^{\circ} \mathrm{C}$. Total protein concentration was determined in the homogenate tissue using a Thermo Scientific Pierce BCA Protein Assay Kit (Thermo Scientific Inc., Hanover Park, IL) after protein extraction in the homogenate. Twelve micrograms of liver homogenate protein extract (30 $\mu \mathrm{L}$ total volume) was resolved in a 4 to $20 \%$ precast polyacrylamide gel (Criterion TGX Stain-Free Precast Gel, $30 \mu \mathrm{L}$; Bio-Rad Laboratories Inc.). The electrophoresis was performed at 250 to $300 \mathrm{~V}$ in a wide vertical electrophoresis tank (Criterion; Bio-Rad Laboratories Inc.) for 25 min. Subsequently, the separated proteins were transferred from the gels onto Trans-Blot Turbo Mini PVDF Transfer Packs (Bio-Rad Laboratories Inc.) with a Trans-Blot Turbo Transfer System (Bio-Rad Laboratories Inc.) for 6 min. The software used (Image Lab Software; BioRad Laboratories Inc.) in this equipment allowed us to quantify the efficiency of the transfer. The yield of the transfer was approximately 99.9\%. Before transferring the membranes, they were incubated in blocking solu- 
tion $\{5 \%$ dry milk in Tris-buffered saline (TBS) with Tween-20 (TTBS) $[0.8 \% \mathrm{ClNa}$ and $0.242 \%$ 2-amino2-(hydroxymethyl)-1,3-propanediol] and 1\% Tween-20 (Bio-Rad Laboratories Inc.) $\}$ for $1 \mathrm{~h}$ at room temperature, washed 3 times in TBS, and finally incubated with primary (DMT1) antibody in TTBS overnight at $4^{\circ} \mathrm{C}$ with gentle and continuous agitation. Immunodetection was performed using a polyclonal antibody for DMT1 (Santa Cruz Biotechnology Inc., Santa Cruz, $\mathrm{CA}$ ) diluted to 1:500 and a monoclonal rabbit antibody specific for $\beta$-actin (Rabm and Abcam) diluted to 1:1200 in TTBS was used as a control for total protein loaded in the blot and it was also used to determine the proportions of complex DMT1 in reference to whole hepatic protein content. Blots were then washed 3 times for 5 min each in TTBS and incubated with the appropriate secondary conjugated antibody [ImmunStar Goat Anti-Mouse (GAM)-HRP and Immun-Star Goat Anti-Rabbit (GAR)-HRP; Bio-Rad Laboratories Inc.; $1: 130,000]$ in TTBS for $1 \mathrm{~h}$ at room temperature. Thereafter, the membranes were washed twice with TTBS for $15 \mathrm{~min}$ and a final wash with TBS was performed for $15 \mathrm{~min}$. Following incubation with the appropriate secondary antibodies, blots were visualized by chemiluminescence. Membrane-bound antibodies were visualized with Clarity Western ECL Substrate (Bio-Rad Laboratories Inc.). Signal quantification and recording for each band were performed with Fujifilm equipment. These results were analyzed with Image $\mathrm{J}$ software (http://rsbweb.nih.gov/ij/). In addition, the total quantity of proteins transferred to the blot was determined with Image Lab Software (Bio-Rad Laboratories Inc.) on the membrane and these quantities were used to standardize all results.

\section{Statistical Analysis}

Statistical analyses were performed using the SPSS computer program (version 21.0, 2012; SPSS Inc., Chicago, IL). All data are reported as mean values with their standard errors. Differences between groups fed normal-Fe- or low-Fe-content diets during the PEP were tested for statistical significance with Student's $t$-test. Variance analysis by one-way ANOVA was used to compare the different diets supplied to the animals. Following a significant F-test $(P<0.05)$, individual means were tested by pairwise comparison with the Tukey multiple comparison test, when main effects and interactions were significant. The level of significance was set at $P<0.05$.

\section{RESULTS AND DISCUSSION}

At the end of the PEP, control rats fed the normal-Fe diet had all the hematological parameters within nor- mal limits for this species. However, the hematological parameters of the anemic rats after feeding them for 40 d with a low-Fe diet were drastically different from the controls $(P<0.001$; Table 2$)$. The key to understanding the measurements of Fe status parameters used in the current study is knowledge of Fe homeostasis. The newly absorbed Fe $(1-2 \mathrm{mg} / \mathrm{d})$ enters the intracellular Fe pool of enterocyte via DMT1; however, the major source of $\mathrm{Fe}(30-40 \mathrm{mg} / \mathrm{d})$ is provided by the macrophages that recycle Fe from senescent RBC. Iron bound to transferrin enters the hepatocytes by endocytosis of the transferrin-transferrin receptor complex. The Fe cycle is completed when the newly formed erythrocytes are returned to the circulation over the next 7 to 10 d. Iron loss is from desquamation, menstruation, and other blood loss (1-2 mg/d; Zimmermann and Hurrell, 2007). Iron deficiency occurs because the rats with normal body Fe stores lose a large portion of body Fe before the hemoglobin concentration decreases (in the first days of Fe deprivation). Blood hemoglobin concentration is the most reliable indicator of anemia at the population level and it is used in national demographic health surveys and by national governments surveying populations before making new policy for nutritional interventions. After the ingestion of aged RBC by macrophages in the spleen and bone marrow, Fe is extracted from hemoglobin and returned to the plasma where it is tightly bound to transferrin. The Fe cycle is completed when the newly formed erythrocytes are returned to the circulation over the next week. Other important communications exist between the plasma Fe compartment and the intestinal mucosa and liver. Serum Fe, TIBC, and transferrin saturation tests are used to screen for and monitor conditions of Fe deficiency and overload. The major source of Fe for transferrin is the reticuloendothelial system, with some from hepatocytes and the intestine. Transferrin saturation can decrease to $5 \%$ in Fe deficiency (Bothwell et al., 1979), as occurred in the current study. Serum transferrin concentrations are derived mostly from developing $\mathrm{RBC}$ and so they reflect the intensity of erythropoiesis and the demand for Fe; the concentration rises in Fe-deficiency anemia and it is a marker of the severity of Fe insufficiency, but only when Fe stores have been exhausted, provided that no other causes of abnormal erythropoiesis exist (WHOCDC, 2007). The serum ferritin level is considered as the best single and noninvasive test for the diagnosis of Fe deficiency because its concentration is proportional to total-body Fe stores. A low ferritin level is pathognomonic of Fe deficiency (Goddard et al., 2011). Finally, serum hepcidin levels are drastically reduced in the anemic group $(P<0.001)$, reflecting the impairment of $\mathrm{Fe}$ status and its reduction is an accurate indicator of Fe deficiency (Pasricha et al., 2011). Therefore, tak- 
Table 2. Hematological parameters of rats fed either normal-Fe or low-Fe diets for $40 \mathrm{~d}^{1}$

\begin{tabular}{lcc}
\hline $\begin{array}{l}\text { Hematological } \\
\text { parameter }\end{array}$ & $\begin{array}{c}\text { Normal-Fe control } \\
\text { group }\end{array}$ & $\begin{array}{c}\text { Low-Fe anemic } \\
\text { group }\end{array}$ \\
\hline Hemoglobin concentration, g/L & $127.12 \pm 2.23$ & $73.48 \pm 2.45^{*}$ \\
RBC, $10^{12} / \mathrm{L}$ & $7.17 \pm 0.14$ & $6.23 \pm 0.15^{*}$ \\
Hematocrit, $\%$ & $40.07 \pm 0.66$ & $28.08 \pm 0.57^{*}$ \\
MCV, $\mathrm{fL}$ & $56.12 \pm 0.23$ & $39.35 \pm 0.52^{*}$ \\
Platelets, $10^{9} / \mathrm{L}$ & $731 \pm 25.02$ & $1,386 \pm 69.12^{*}$ \\
Serum Fe, $\mu \mathrm{g} / \mathrm{L}$ & $1,378 \pm 118$ & $698 \pm 59^{*}$ \\
TIBC, $\mu \mathrm{g} / \mathrm{L}$ & $2,775 \pm 198$ & $18,022 \pm 687^{*}$ \\
Transferrin saturation, $\%$ & $48.13 \pm 6.55$ & $3.92 \pm 0.43^{*}$ \\
Serum ferritin, $\mu \mathrm{g} / \mathrm{L}$ & $81.97 \pm 2.57$ & $51.05 \pm 1.53^{*}$ \\
Serum hepcidin, $\mathrm{ng} / \mathrm{mL}$ & $17.01 \pm 0.44$ & $13.23 \pm 0.6^{*}$ \\
\hline
\end{tabular}

${ }^{1}$ Mean value \pm SEM for 30 rats per group.

${ }^{2} \mathrm{RBC}=$ red blood cells; $\mathrm{MCV}=$ mean corpuscular volume; $\mathrm{TIBC}=$ total Fe-binding capacity.

*Significantly different $(P<0.001)$ from the control group by Student's $t$-test.

ing into account all of that mentioned above, we can conclude that the degree of Fe deficiency was severe and the body stores were deeply depleted after dietary Fe restriction (Table 2).

At the end of the EP, most of the hematological parameters were recovered with both milk-based diets; however, the platelet concentrations still were elevated $(P<0.001)$ and the serum ferritin and hemoglobin concentrations were low $(P<0.01$ for ferritin and $P<0.05$ for hemoglobin) in the anemic group fed the cow milkbased diet compared with its controls, revealing that Fe repletion was more efficient with goat milk (Table 3 ). This fact can be explained due to the beneficial nutritional characteristics of goat milk compared with cow milk: caprine milk contains a higher proportion of soluble proteins ( $\beta-\mathrm{LG}, \alpha-\mathrm{LA}$, and serum albumin), which favors $\mathrm{Fe}$ absorption and a higher amount of dietary components capable of reducing $\mathrm{Fe}$ (III) to Fe (II), including ascorbic acid and AA such as cysteine, forming chelates with $\mathrm{Fe}$, which remain soluble at a higher $\mathrm{pH}$ within the small intestine (Alférez et al., 2006). In addition, the RBC count and serum hepcidin concentration were higher in both groups fed the goat milk diet (RBC count: $P<0.01$ for control and $P<$ 0.001 for anemic rats; serum hepcidin: $P<0.001$ for control and $P<0.01$ for anemic rats) (Table 3 ). The higher expression of DMT1 (Figure 1A and B) and the higher amount of serum hepcidin found in the current study support the better Fe storage and, therefore, the recovery of the hematological parameters after $30 \mathrm{~d}$ of supplying goat milk.

The BW at d 40 was lower in anemic animals $(P<$ 0.001 ), and the hepatic weight also decreased slightly. As a consequence, the hepatosomatic index (ratio of liver weight to $\mathrm{BW}$ ) was higher in the Fe-deficient animals $(P<0.01$; Table 4$)$. On the other hand, the degree of Fe deficiency produced by the Fe-restrictive $\operatorname{diet}(\mathrm{PEP})$ was severe enough to impair the $\mathrm{BW}$, which coincides with the results of other authors (Strube et al., 2002) and it is possible to attribute this fact to the lower levels of thyroid hormones found in this pathology (Beard et al., 1998). Moreover, as mentioned above, the hemoglobin and RBC count diminished drastically and the supply of oxygen to the cells limited itself considerably, which affected in a negative way the ATP synthesis and BW (Schneider et al., 2008). However, at the end of the EP, because the rats completely recovered from the anemia and had an increased supply of oxygen to the cells, which improved the ATP synthesis and weight gain, no differences were found in the BW or hepatosomatic indexes with the milk-based diets assayed.

The differences in the Fe hepatic content on d $40(P$ $<0.001$ ) reveals that the $\mathrm{Fe}$ deposits were deeply depleted and a severe degree of Fe deficiency was reached, induced by the consumption of a restricted-Fe diet for $40 \mathrm{~d}$. The lower $\mathrm{Fe}$ content recorded in the liver in the situation of Fe-deficiency anemia can be due to the decrease in the serum hepcidin concentration recorded in the current study. Studies of Fe deficiency in hepcidin knockout mice suggest that this peptide intervenes regulating the storage of the mineral (attenuating both the intestinal Fe absorption and the liberation of the Fe of the macrophages and hepatocytes). On the other hand, a low expression of hepcidin in the situation of Fe overload suggests that hepcidin is also a factor in erythroid regulation, showing diminished expression in response to anemic hypoxia (Zhang and Enns, 2009). Therefore, severe Fe deficiency, such as that induced in the current study, correlates with lower hepcidin levels, although this may be modulated by induction of erythropoiesis (which also reduces hepcidin levels; Nemeth and Ganz, 2009). In addition, hepcidin also controls the Fe levels directly acting reciprocally with ferroportin 1, driving the internalization and degradation of FPN1 when levels of Fe are high and consequently blocking 
Table 3. Hematological parameters of control and anemic rats fed goat or cow milk-based diets with normal Fe content for $30 \mathrm{~d}^{1}$

\begin{tabular}{lccccc}
\hline & \multicolumn{2}{c}{ Goat milk-based diet } & & \multicolumn{2}{c}{ Cow milk-based diet } \\
\cline { 2 - 3 } \cline { 5 - 6 } $\begin{array}{l}\text { Hematological } \\
\text { parameter }\end{array}$ & Control & Anemic & & Control & Anemic \\
\hline Hb concentration, g/L & $130.8 \pm 3.9$ & $132.5 \pm 3.4^{\mathrm{A}}$ & & $127.6 \pm 4.3$ & $120.8 \pm 2.1^{\mathrm{B}, \mathrm{d}}$ \\
RBC, $10^{12} / \mathrm{L}$ & $7.79 \pm 0.24^{\mathrm{a}}$ & $7.71 \pm 0.28^{\mathrm{A}}$ & & $7.08 \pm 0.27^{\mathrm{b}}$ & $6.98 \pm 0.26^{\mathrm{B}}$ \\
Hematocrit, $\%$ & $42.78 \pm 1.42$ & $40.14 \pm 0.95$ & & $40.82 \pm 1.48$ & $38.4 \pm 1.99$ \\
MCV, fL & $57.25 \pm 0.50$ & $55.04 \pm 1.17$ & & $57.68 \pm 0.82$ & $55.34 \pm 1.28$ \\
Platelets, $10^{9} / \mathrm{L}$ & $814.21 \pm 41.43$ & $784.00 \pm 35.55^{\mathrm{A}}$ & & $826.00 \pm 43.45$ & $939.33 \pm 35.30^{\mathrm{B}, \mathrm{d}}$ \\
Serum Fe, $\mu \mathrm{g} / \mathrm{L}$ & $1,410 \pm 119$ & $1,375 \pm 101^{\mathrm{A}}$ & & $1,385 \pm 118$ & $1,209 \pm 98^{\mathrm{B}}$ \\
TIBC, $\mu \mathrm{g} / \mathrm{L}$ & $2,703 \pm 203$ & $2,725 \pm 203$ & & $2,795 \pm 195$ & $2,923 \pm 221$ \\
Transferrin saturation, $\%$ & $46.13 \pm 4.98$ & $45.42 \pm 5.12$ & & $45.31 \pm 5.31$ & $42.13 \pm 3.97$ \\
Serum ferritin, $\mu \mathrm{g} / \mathrm{L}$ & $80.03 \pm 2.25$ & $78.95 \pm 2.01^{\mathrm{A}}$ & & $78.43 \pm 2.77$ & $70.01 \pm 1.97^{\mathrm{B}, \mathrm{d}}$ \\
Serum hepcidin, ng/mL & $17.10 \pm 0.53^{\mathrm{a}}$ & $14.30 \pm 0.70^{\mathrm{A}, \mathrm{d}}$ & & $14.31 \pm 0.95^{\mathrm{b}}$ & $12.27 \pm 0.90^{\mathrm{B}}$ \\
\hline
\end{tabular}

$\overline{\mathrm{a}, \mathrm{b}}$ Mean values within a row and within control groups with different superscript lowercase letters differ $(P<$ $0.05)$ by Student's $t$-test.

${ }^{\mathrm{A}, \mathrm{B}}$ Mean values within a row and within anemic groups with different superscript capital letters differ $(P<$ $0.05)$ by Student's $t$-test.

${ }^{\mathrm{d}}$ Indicates difference $(P<0.05)$ for control vs. anemic group within a diet by the Tukey test.

${ }^{1}$ Mean value \pm SEM for 10 rats per group.

${ }^{2} \mathrm{Hb}=$ hemoglobin; $\mathrm{RBC}=$ red blood cells; $\mathrm{MCV}=$ mean corpuscular volume; TIBC $=$ total Fe-binding capacity.

the liberation of the Fe in the sites of storage (Nemeth and Ganz, 2009). In this sense, the increase in serum hepcidin concentration explains the higher liver $\mathrm{Fe}$ content after supplying goat milk for $30 \mathrm{~d}$ in anemic animals, because the higher levels of this peptide hor-

\section{(A)}

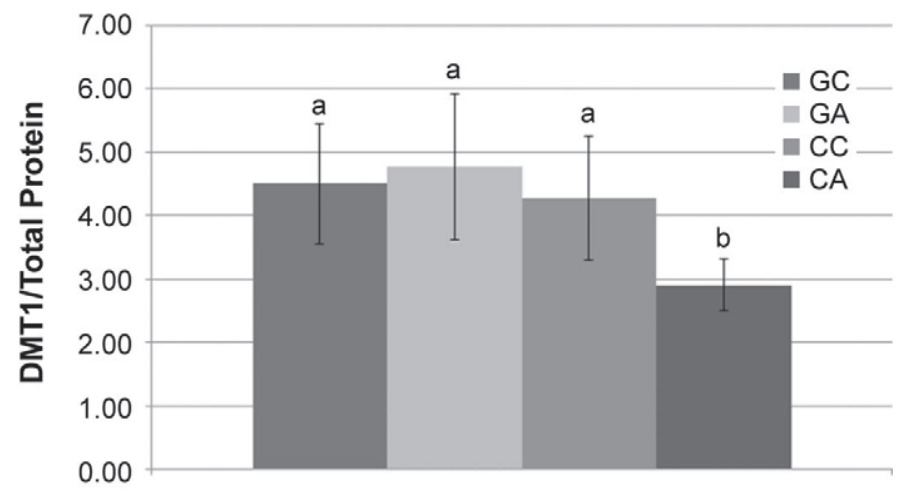

(B)

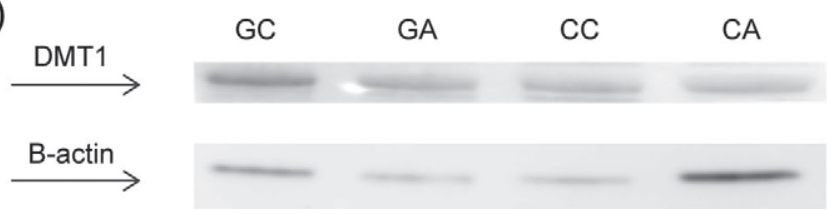

Figure 1. Effect of goat or cow milk-based diet consumption on hepatic expression of divalent metal transporter 1 (DMT1) in control and anemic rats (A). Representative immunoblots of DMT1 and B-actin are shown $(B)$. Values are means \pm SEM $(\mathrm{n}=10)$. Within a protein, means without a common letter $(\mathrm{a}$ and $\mathrm{b})$ differ $(P<0.05)$. $\mathrm{GC}=$ control rats fed a goat milk diet; $\mathrm{GA}=$ anemic rats fed a goat milk diet; $\mathrm{CC}=$ control rats fed a cow milk diet; $\mathrm{CA}=$ anemic rats fed a cow milk diet. mone compared with the cow milk diet decreases the Fe export from hepatocytes, increasing its storage in the liver. The Fe content in the liver was lower in both groups of animals fed cow milk $(P<0.01$ for control and $P<0.001$ for anemic rats; Table 5).

We analyzed the expression of DMT1 in control and anemic rats after consumption of cow or goat milk-based diets to confirm the homeostatic response to dietary Fe. In the liver, DMT1 was differentially expressed in response to the milk-based diets, being significantly increased in anemic rats fed goat milk compared with cow milk $(P<0.01$; Figure $1 \mathrm{~A}$ and $\mathrm{B})$. The DMT1 expression of control and anemic rats fed goat milk was respectively 105.99 and $145.26 \%$ of the DMT1 expression of rats fed cow milk $(P<0.05)$.

Divalent metal transporter 1 is localized to both the plasma membrane and an intracellular compartment in hepatocytes (Trinder et al., 2000). In addition to its role in endosomal Fe export, the presence of DMT1 on the microvillous membrane of hepatocytes suggests

Table 4. Body weight, liver weight, hepatosomatic index, and liver Fe content in rats fed either normal-Fe or low-Fe diets for $40 \mathrm{~d}^{1}$

\begin{tabular}{lcc}
\hline Index & $\begin{array}{c}\text { Control } \\
\text { group }\end{array}$ & $\begin{array}{c}\text { Anemic } \\
\text { group }\end{array}$ \\
\hline BW, g & $298.4 \pm 7.8$ & $270.2 \pm 7.5^{*}$ \\
Liver weight, g & $7.926 \pm 0.25$ & $7.598 \pm 0.33$ \\
Liver weight/BW, \% & $2.60 \pm 0.06$ & $2.79 \pm 0.09^{*}$ \\
Liver Fe, $\mu \mathrm{g} / \mathrm{g}$ of DM & $495.35 \pm 31.88$ & $309.91 \pm 28.35^{*}$ \\
\hline
\end{tabular}

${ }^{1}$ Mean value \pm SEM for 10 rats per group.

*Significantly different $(P<0.05)$ from the control group by Student's t-test. 
Table 5. Body weight, liver weight, hepatosomatic index, and liver Fe content in control and anemic rats fed goat or cow milk-based diets with normal Fe content for $30 \mathrm{~d}^{1}$

\begin{tabular}{lccccc}
\hline & \multicolumn{2}{c}{ Goat milk-based diet } & & \multicolumn{2}{c}{ Cow milk-based diet } \\
\cline { 2 - 3 } \cline { 5 - 6 } Parameter & Control & Anemic & & Control & Anemic \\
\hline BW, g & $301.00 \pm 6.73$ & $289.65 \pm 6.19$ & & $305.27 \pm 7.55$ & $288.25 \pm 7.32$ \\
Liver weight, g & $7.69 \pm 0.13$ & $7.45 \pm 0.15$ & & $7.72 \pm 0.20$ & $7.31 \pm 0.20$ \\
Liver weight/BW, $\%$ & $2.56 \pm 0.09$ & $2.57 \pm 0.08$ & & $2.52 \pm 0.08$ & $2.51 \pm 0.07$ \\
Liver Fe, $\mu \mathrm{g} / \mathrm{g}$ of $\mathrm{DM}$ & $623.10 \pm 35.22^{\mathrm{a}}$ & $562.04 \pm 33.14^{\mathrm{A}, \mathrm{d}}$ & & $552.93 \pm 31.31^{\mathrm{b}}$ & $398.81 \pm 29.28^{\mathrm{B}, \mathrm{d}}$ \\
\hline
\end{tabular}

${ }^{a, b}$ Mean values within a row and within control groups with different superscript lowercase letters differ $(P<$ $0.05)$ by Student's $t$-test.

${ }^{\mathrm{A}, \mathrm{B}}$ Mean values within a row and within anemic groups with different superscript capital letters differ $(P<$ $0.05)$ by Student's $t$-test.

${ }^{\mathrm{d}}$ Indicates difference $(P<0.05)$ for control vs. anemic group within a diet by the Tukey test.

${ }^{1}$ Mean value \pm SEM for 10 rats per group.

that the liver absorbs non-heme, non-transferrin-bound Fe from the circulation. Unlike intestine DMT1, hepatic DMT1 expression parallels Fe status (Trinder et al., 2000), suggesting that DMT1 plays a key role in cellular protection from excess Fe as opposed to regulating $\mathrm{Fe}$ homeostasis in the liver. Therefore, the increased liver DMT1 protein levels reflect faithfully the increased liver $\mathrm{Fe}$ storage after Fe repletion (Kelleher and Lönnerdal, 2005) and, therefore, the increase in the DMT1 level in anemic animals fed goat milk reveals that Fe repletion after induced anemia is more efficient when supplying goat milk, a finding that is supported and corroborated by the higher Fe storage in the rats fed goat milk compared with cow milk.

On the other hand, we have previously reported that goat milk improves Ca metabolism, due to its major content of vitamin $\mathrm{D}$, which increases Ca energy-dependent transcellular transport and has higher lysine and cysteine content, nutrients that improve $\mathrm{Ca}$ absorption via passive transport (Alférez et al., 2006). Moreover, Díaz-Castro et al. (2011) reported that goat milk provoked a decrease in the rate of parathormone secretion, improving Ca utilization and its deposit in target organs and that all these factors would lead to high efficiency in the DMT1 Fe-transport activity. Elevated intracellular Ca concentration has been reported to increase non-transferrin-bound Fe uptake through an undefined transport system that has characteristics distinct from DMT1 (Kaplan et al., 1991) and, in addition, Ca has an important and ubiquitous role in cell signaling. An adequate $\mathrm{Ca}$ homeostasis ameliorates the Fe-transport defect caused by inactivation of DMT1 in microcytic $(m k)$ mice, either in the intestine, liver, or in erythroid precursors (Xu et al., 2004). The transferrin cycle is essential for Fe uptake by erythroid precursor cells, and DMT1 mediates the transfer of Fe from transferrin cycle endosomes to the cytoplasm (Fleming et al., 1998; Touret et al., 2003). Elevated Ca has been reported to accelerate Fe uptake through the transferrin cycle, apparently through activation of protein kinase $\mathrm{C}(\mathrm{Ci}$ et al., 2003). All these results add an interesting perspective to the emerging role of Ca for DMT-1 function (Gunshin et al., 1997). Thus, in light of these considerations, we can hypothesize that the improvement in $\mathrm{Ca}$ metabolism reported with goat milk might potentiate the liver DMT1 Fe-transport activity and expression, increasing Fe storage, supporting and explaining the results of the current study.

In addition, goat milk fat is richer in medium-chain triglycerides, which are oxidized in the mitochondria, providing fast energy discharge that can be used in several metabolic pathways (Alférez et al., 2006) and thus contribute to increasing the synthesis of carrier proteins such as DMT1 and, hence, the absorption of Fe. On the other hand, a low-vitamin A diet reduced liver DMT1 protein expression by posttranscriptional regulation via decreased protein translation or increased degradation, negatively affecting Fe metabolism (Kelleher and Lönnerdal, 2005). In this sense, goat milk would increase the expression of DMT1, because it has more than twice the vitamin A content of cow milk (Alférez et al., 2006)

\section{CONCLUSIONS}

During the recovery from anemia with goat or cow milk-based diets, the restoration of liver $\mathrm{Fe}$ content and hematological parameters increases the supply of oxygen to the cells, favoring weight gain. Moreover, goat milk consumption potentiates the liver DMT1 Fe-transport activity and expression, enhancing $\mathrm{Fe}$ metabolism and storage compared with cow milk. In addition, the increase in serum hepcidin concentration in anemic rats also explains and supports the higher liver Fe content after supplying goat milk, because it blocks the liberation of Fe from hepatocytes, increasing its storage in liver. Thus, dietary modulation of DMT1 function and serum hepcidin by goat milk consumption in rats modulates whole-body Fe homeostasis, improv- 
ing hematological parameters and Fe storage in liver, positively affecting Fe metabolism in Fe-deficiency anemia, compared with cow milk.

\section{ACKNOWLEDGMENTS}

This work was supported by Junta de Andalucía Excellence research project No. P11-AGR-7648. The authors are grateful to Lactalis Iberia S.A. (Lugo, Spain) for providing the dairy products used in this study, Elisa Alcover for her efficient administrative support, and Encarnación Rebollo for her competent technical assistance (both from Department of Physiology, University of Granada, Spain).

\section{REFERENCES}

Alférez, M. J. M., I. López-Aliaga, T. Nestares, J. Díaz-Castro, M. Barrionuevo, P. B. Ros, and M. S. Campos. 2006. Dietary goat milk improves iron bioavailability in rats with induced nutritional ferropenic anemia in comparison with cow milk. Int. Dairy J. 16:813-821.

Anderson, G. J., and C. D. Vulpe. 2009. Mammalian iron transport. Cell. Mol. Life Sci. 66:3241-3261.

Beard, J. L., D. E. Brigham, S. K. Kelley, and M. H. Green. 1998 Plasma thyroid hormone kinetics are altered in iron-deficient rats. J. Nutr. 128:1401-1408.

Bothwell, T. H., R. W. Charlton, J. D. Cook, and C. A. Finch. 1979. Iron Metabolism in Man. Blackwell Scientific Publications, Oxford, UK.

Ci, W., W. Li, Y. Ke, Z.-M. Qian, and X. Shen. 2003. Intracellular $\mathrm{Ca}^{2+}$ regulates the cellular iron uptake in K562 cells. Cell Calcium $33: 257-266$.

De Domenico, I., D. M. Ward, C. Langelier, M. B. Vaughn, E. Nemeth, W. I. Sundquist, T. Ganz, G. Musci, and J. Kaplan. 2007. The molecular mechanism of hepcidin-mediated ferroportin downregulation. Mol. Biol. Cell 18:2569-2578.

Díaz-Castro, J., M. J. M. Alférez, I. López-Aliaga, T. Nestares, S. Granados, M. Barrionuevo, and M. S. Campos. 2008. Influence of nutritional iron deficiency anemia on DNA stability and lipid peroxidation in rats. Nutrition 24:1167-1173.

Díaz-Castro, J., M. Ramírez López-Frías, M. S. Campos, M. LópezFrías, M. J. M. Alférez, T. Nestares, E. Ortega, and I. López-Aliaga. 2011. Goat milk during iron repletion improves bone turnover impaired by severe iron deficiency. J. Dairy Sci. 94:2752-2761.

Ekström, E. C., S. M. Hyder, A. M. Chowdhury, S. A. Chowdhury, B. Lönnerdal, J. P. Habicht, and L. A. Persson. 2002. Efficacy and trial effectiveness of weekly and daily iron supplementation among pregnant women in rural Bangladesh: Disentangling the issues. Am. J. Clin. Nutr. 76:1392-1400.

Fleming, M. D., M. A. Romano, M. A. Su, L. M. Garrick, M. D. Garrick, and N. C. Andrews. 1998. Nramp2 is mutated in the anemic Belgrade (b) rat: Evidence of a role for Nramp2 in endosomal iron transport. Proc. Natl. Acad. Sci. USA 95:1148-1153.

Ganz, T. 2011. Hepcidin and iron regulation, 10 years later. Blood 117:4425-4433.

Goddard, A. F., M. W. James, A. S. McIntyre, and B. B. Scott. 2011. Guidelines for the management of iron deficiency anemia. Gut 60:1309-1316

Grootveld, M., J. D. Bell, B. Halliwell, O. I. Aruoma, A. Bomford, and P. J. Sadler. 1989. Non-transferrin-bound iron in plasma or serum from patients with idiopathic hemochromatosis. Characterization by high performance liquid chromatography and nuclear magnetic resonance spectroscopy. J. Biol. Chem. 264:4417-4422.

Gunshin, H., Y. Fujiwara, A. O. Custodio, C. Direnzo, S. Robine, and N. C. Andrews. 2005. Slc11a2 is required for intestinal iron absorp- tion and erythropoiesis but dispensable in placenta and liver. J. Clin. Invest. 115:1258-1266.

Gunshin, H., B. Mackenzie, U. V. Berger, Y. Gunshin, M. F. Romero, W. F. Boron, S. Nussberger, J. L. Gollan, and M. A. Hediger. 1997. Cloning and characterization of a mammalian proton-coupled metal-ion transporter. Nature 388:482-488.

Kaplan, J., I. Jordan, and A. Sturrock. 1991. Regulation of the transferrin-independent iron transport system in cultured cells. J. Biol. Chem. 266:2997-3004.

Kelleher, S. L., and B. Lönnerdal. 2005. Low vitamin A intake affects milk iron level and iron transporters in rat mammary gland and liver. J. Nutr. 135:27-32.

Nemeth, E., and T. Ganz. 2009. The role of hepcidin in iron metabolism. Acta Haematol. 122:78-86.

Nestares, T., M. Barrionuevo, J. Díaz-Castro, I. López-Aliaga, M. J. M. Alférez, and M. S. Campos. 2008. Calcium enriched goats' milk aids recovery of iron status better than calcium-enriched cows milk, in rats with nutritional ferropenic anaemia. J. Dairy Res. $75: 153-159$.

Pallarés, I., F. Lisbona, I. López-Aliaga, M. Barrionuevo, M. J. M. Alférez, and M. S. Campos. 1993. Effects of iron deficiency on the digestive utilization of iron, phosphorus, calcium and magnesium in rats. Br. J. Nutr. 70:609-620.

Pasricha, S.-R., Z. K. McQuilten, M. Westerman, A. Keller, E. Nemeth, T. Ganz, and E. M. Wood. 2011. Serum hepcidin as a diagnostic test of iron deficiency in premenopausal female blood donors. Haematologica 96:1099-1105.

Pietrangelo, A. 2010. Hereditary hemochromatosis: Pathogenesis, diagnosis, and treatment. Gastroenterology 139:393-408.

Reeves, P. G., F. H. Nielsen, and G. C. Fahey Jr.. 1993. AIN-93 purified diets for laboratory rodents: Final report of the American Institute of Nutrition and Ad Hoc Writing Committee on the reformulation of the AIN-76A rodent diet. J. Nutr. 123:1939-1951.

Schneider, J. M., M. L. Fujii, C. L. Lamp, B. Lönnerdal, K. G. Dewey and S. Zidenberg-Cherr. 2008. The use of multiple logistic regression to identify risk factors associated with anemia and iron deficiency in a convenience sample of 12-36-mo-old children from low-income families. Am. J. Clin. Nutr. 87:614-620.

Strube, Y. N. J., J. L. Beard, and A. C. Ross. 2002. Iron deficiency and marginal vitamin A deficiency affect growth hematological indices and the regulation of iron metabolism genes in rats. J. Nutr. 132:3607-3615.

Touret, N., W. Furuya, J. Forbes, P. Gros, and S. Grinstein. 2003 Dynamic traffic through the recycling compartment couples the metal transporter Nramp2 (DMT1) with the transferrin receptor. J. Biol. Chem. 278:25548-25557.

Trinder, D., P. S. Oates, C. Thomas, J. Sadleir, and E. H. Morgan. 2000. Localisation of divalent metal transporter 1 (DMT1) to the microvillus membrane of rat duodenal enterocytes in iron deficiency, but to hepatocytes in iron overload. Gut 46:270-276.

Tselepis, C., S. J. Ford, A. T. McKie, W. Vogel, H. Zoller, R. J. Simpson, J. Diaz Castro, T. H. Iqbal, and D. G. Ward. 2010. Characterization of the transition-metal-binding properties of hepcidin. Biochem. J. 427:289-296.

Viatte, L., and S. Vaulont. 2009. Hepcidin, the iron watcher. Biochimie 91:1223-1228.

WHO (World Health Organization). 2008. Worldwide prevalence of anemia 1993-2005. In WHO Global Database on Anemia. Bruno de Benoist, Erin McLean, Ines Egli, Mary Cogswell. WHO, Geneva, Switzerland.

WHO-CDC (World Health Organization, Centers for Disease Control and Prevention). 2007. Assessing the Iron Status of Populations. 2nd ed. WHO, Geneva, Switzerland.

Xu, H., J. Jin, L. J. DeFelice, N. C. Andrews, and D. E. Clapham. 2004. A spontaneous, recurrent mutation in divalent metal transporter-1 exposes a calcium entry pathway. PLoS Biol. 2:E50.

Zhang, A.-S., and C. A. Enns. 2009. Iron homeostasis: Recently identified proteins provide insight into novel control mechanisms. J. Biol. Chem. 284:711-715.

Zimmermann, M. B., and R. F. Hurrell. 2007. Nutritional iron deficiency. Lancet 370:511-520. 